\title{
Incorporation of $\mathrm{ZnO} / \mathrm{bioactive}$ glass nanoparticles into alginate/chitosan composite hydrogels for wound closure
}

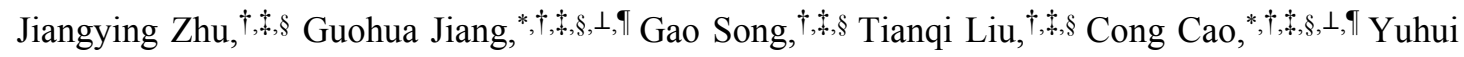
Yang, $,^{\dagger}, \S, \perp, \uparrow$ Yajing Zhang, ${ }^{\dagger}$ and Wenjie Hong ${ }^{\dagger}$

${ }^{\dagger}$ Department of Polymer Materials, College of Materials Science and Engineering, Zhejiang Sci-Tech University, Hangzhou, Zhejiang 310018, China

* National Engineering Laboratory for Textile Fiber Materials and Processing Technology (Zhejiang), Hangzhou 310018, China

$\S$ Key Laboratory of Advanced Textile Materials and Manufacturing Technology (ATMT), Ministry of Education, Hangzhou, Zhejiang 310018, China

$\perp$ Zhejiang-Mauritius Joint Research Center for Biomaterials and Tissue Engineering, Zhejiang Sci-Tech University, Hangzhou, Zhejiang 310018, China

`Institute of Smart Fiber Materials, Zhejiang Sci-Tech University, Hangzhou, Zhejiang 310018, China

Corresponding author: Guohua Jiang, E-mail: ghjiang_cn@zstu.edu.cn, 
Cong Cao, E-mail: cong_zstu@126.com,

\section{Experimental Process for the Evaluation Antibacterial Properties}

To observe the antibacterial capability of the hydrogel more intuitively, the inhibition zone method was utilized. ${ }^{1}$ The Gel-ZBG was placed on the LB agar which was seeded with E. coli and S. aureus bacterial, respectively, and then incubated at $37^{\circ} \mathrm{C}$ for $24 \mathrm{~h}$. Gel was taken as control, and a digital camera was used to record inhibition zone. To evaluate the antibacterial activity of hydrogels in aqueous solution, about 200mg of hydrogel sample was immersed in $4 \mathrm{~mL}$ of the bacterial suspension and incubated in a shaking incubator at $37^{\circ} \mathrm{C}, 100 \mu \mathrm{L}$ of bacteria solution was separately added to 96 -well plate at 15, 30, 60, 120 and $240 \mathrm{~min}$, respectively. The optical density of bacterial suspension was measured at $600 \mathrm{~nm}$ using Enzyme-linked immune detector (Spectra Max Plus 384, Molecular Devices, USA). ${ }^{2,3}$

\section{Experimental Process for the Evaluation the Cytotoxicity}

$1 \times 10^{6}$ cells uniformly dispersed in $200 \mu \mathrm{L}$ of OAL in DMEM were seeded in a 24 -well plate. Then, $300 \mu \mathrm{L}$ of a mixed DMEM medium of SCS and ZBG was added, stirred uniformly, and allowed to stand for a few minutes to obtain Gel-ZBG-cell. Three-dimensional culture of cells 
of ordinary hydrogel (Gel) was used as a control group. The cells inside the hydrogels were subjected to live death staining using FDA-PI staining for 0, 24 and 48 hours in humidified 5\% $\mathrm{CO}_{2} / 95 \%$ air at $37{ }^{\circ} \mathrm{C} .{ }^{4}$ Thereafter, cell reproduction, survival, and death were observed by laser confocal (C2, Nikon, Japan)

\section{Inhibition Zone of BG and ZBG}

The antibacterial effect of BG and ZBG was detected by the Inhibition zone method. ${ }^{5}$ The circular paper sheets having a diameter of $5 \mathrm{~mm}$ were respectively immersed in a $10 \mathrm{mg} / \mathrm{mL} \mathrm{BG}$ or ZBG PBS solution for 24 hours, and then the sheets were plated on agar medium seeded with $1 \times 10^{8} \mathrm{CFU} / \mathrm{ml}$ of $S$. aureus.
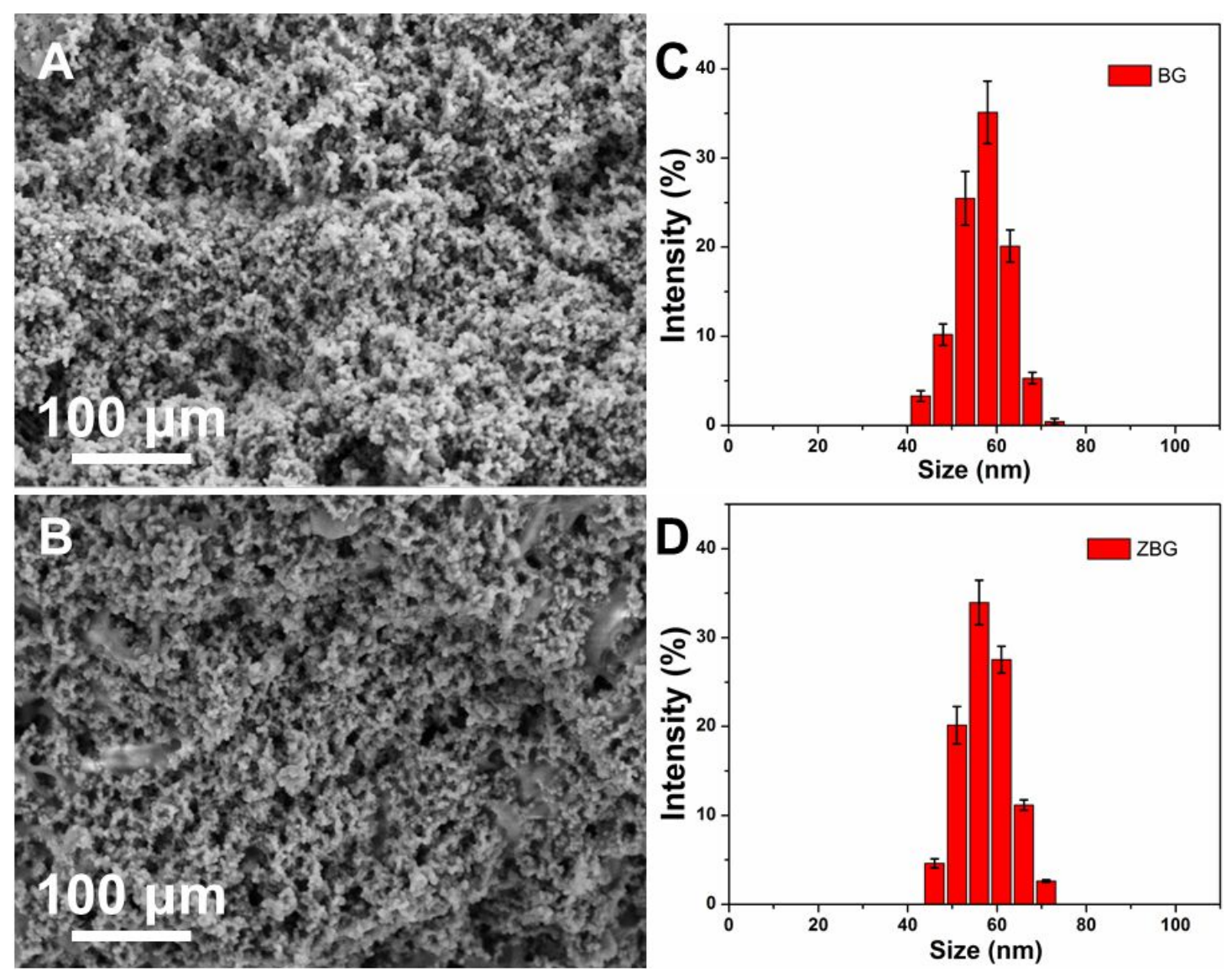
Fig. S1 SEM images of BG (A) and ZBG (B), size distribution of BG (C) and ZBG (D).

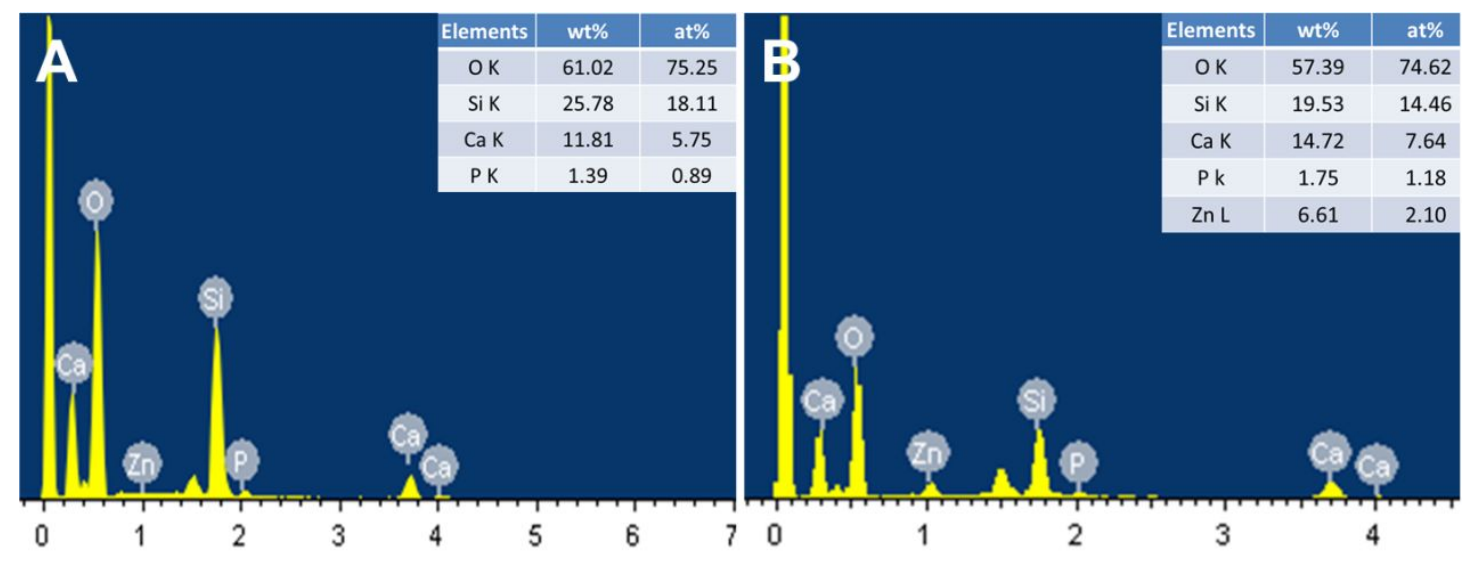

Fig. S2 EDS analysis for BG (A) and ZBG (B).
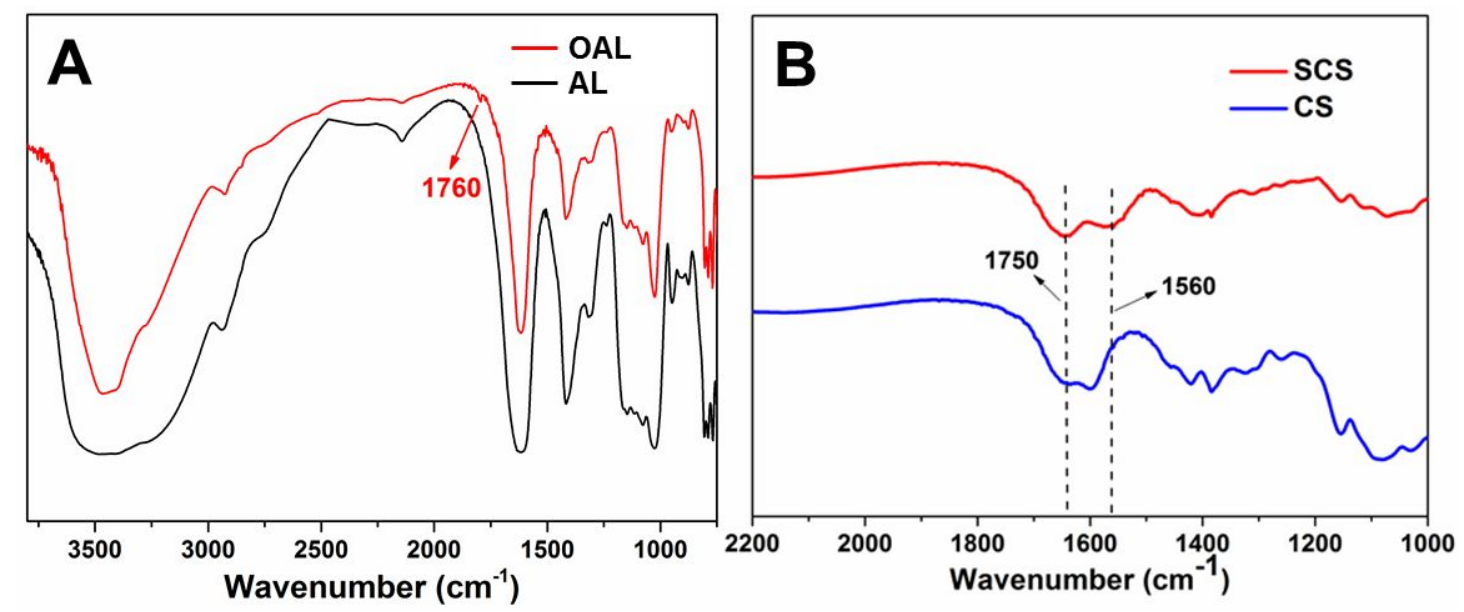

Fig. S3 FT-IR spectra of OAL (A) and SCS (B). 


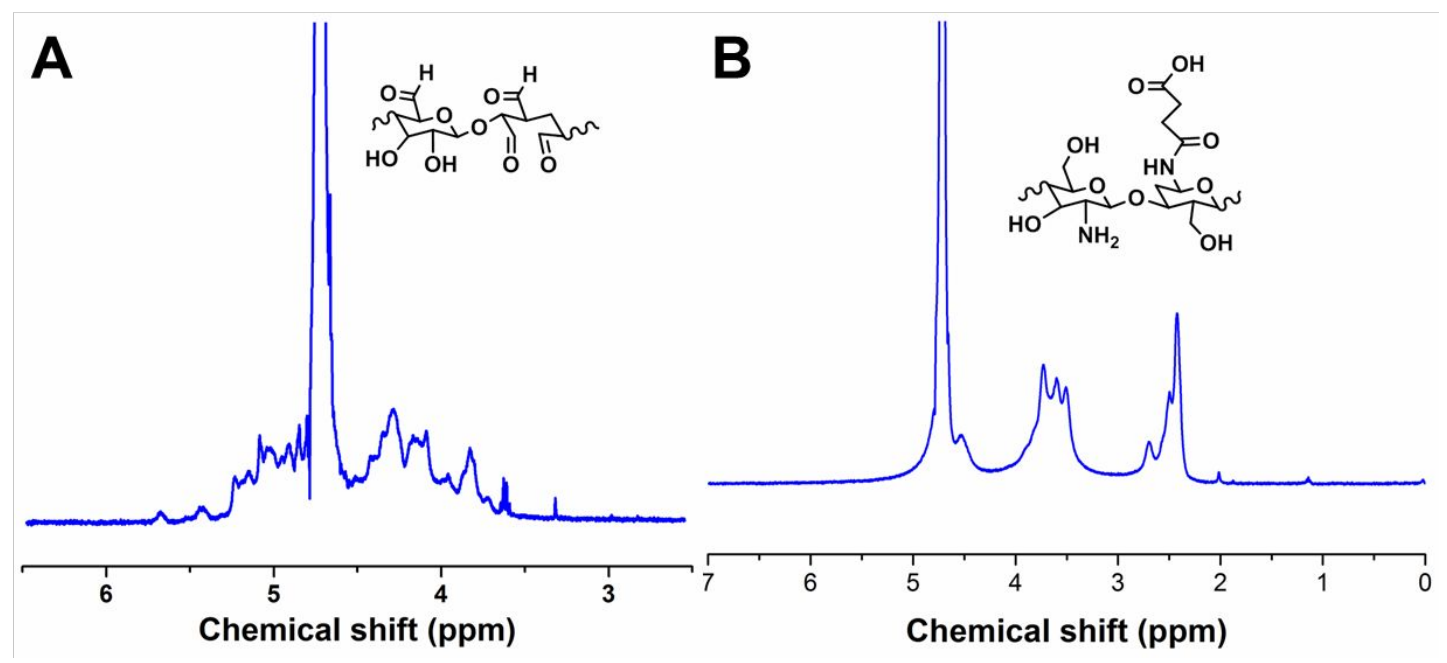

Fig. S4 ${ }^{1} \mathrm{H}$ NMR spectra of OAL (A) and SCS (B).

A

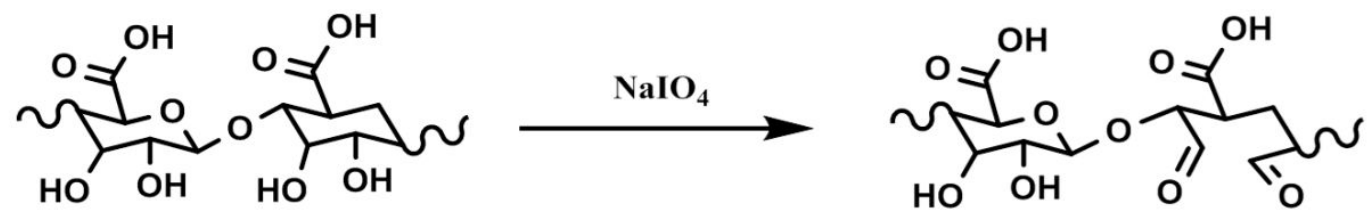

B

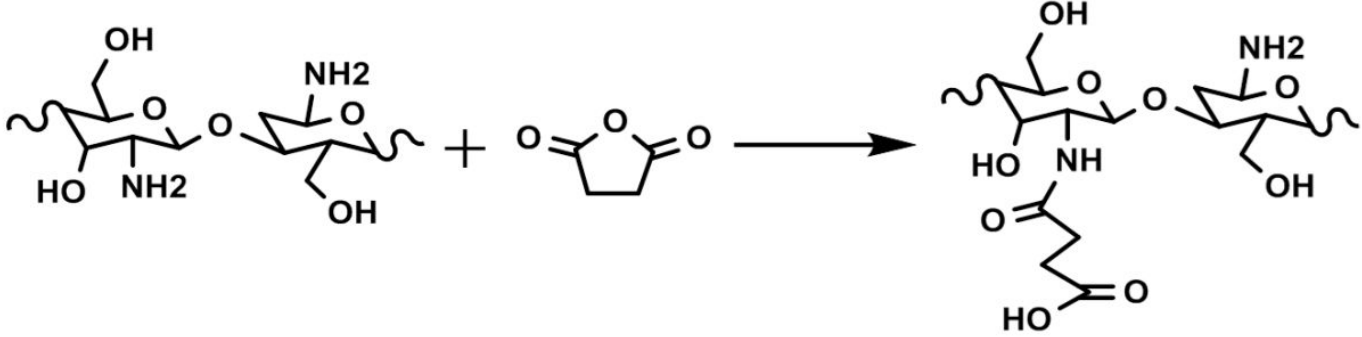

C

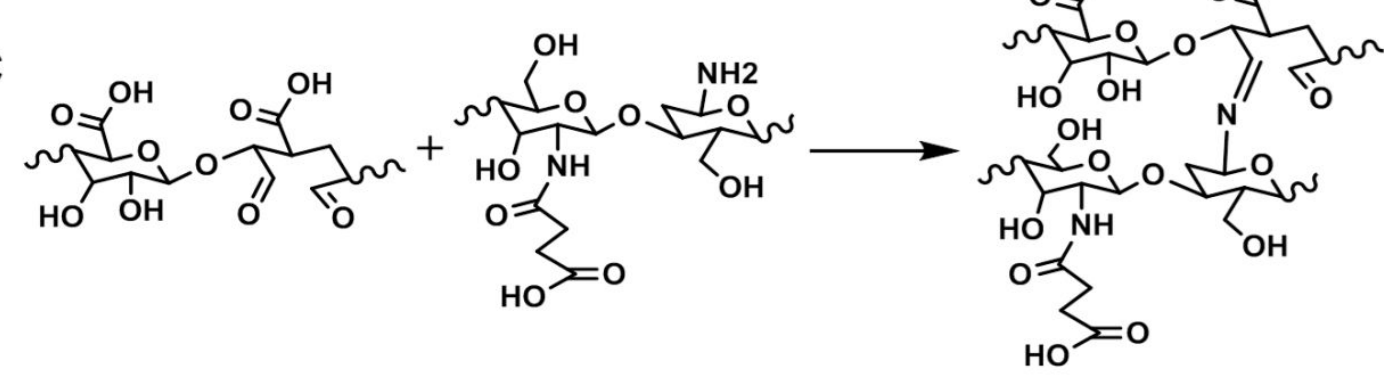

Scheme S1. The synthetic route of OAL (A), SCS (B), and Gel (C). 


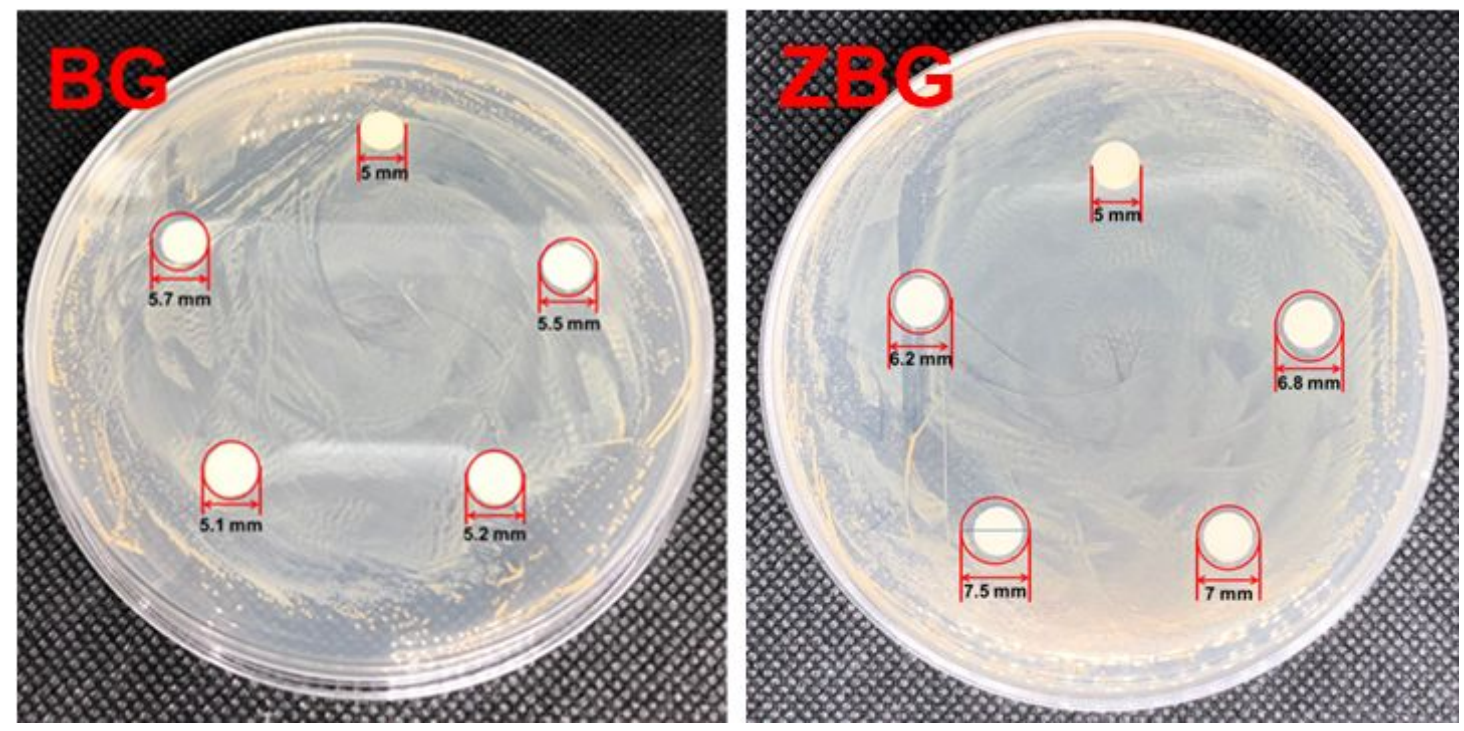

Fig. S5 Optical images of BG and ZBG against the inhibition zone of and S. aureus.

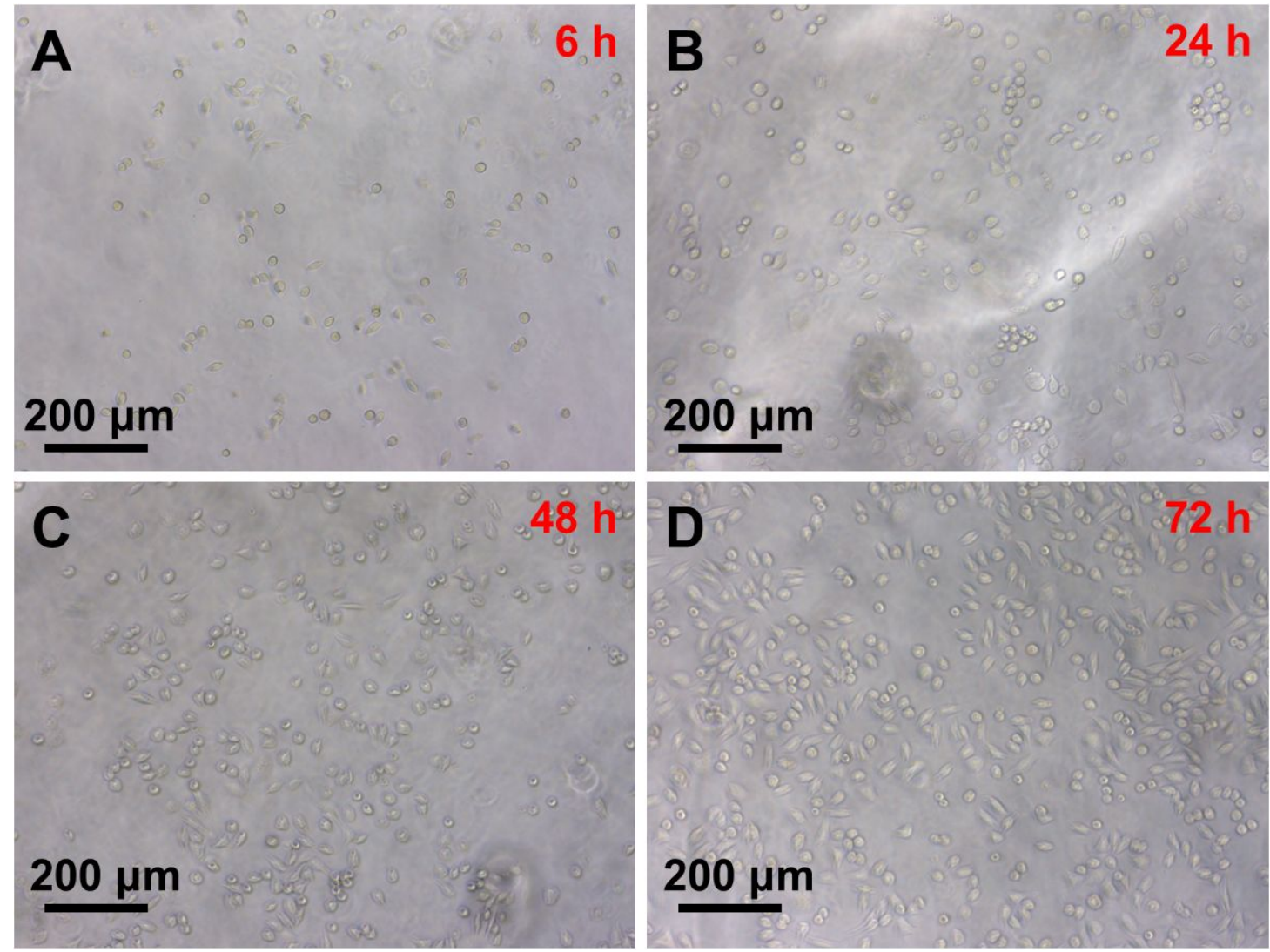

Fig. S6 Micrographs of L929 cells after incubation for 6 (A), 24 (B), 48 (C) and 72 h (D) on 
surface of hydrogels.
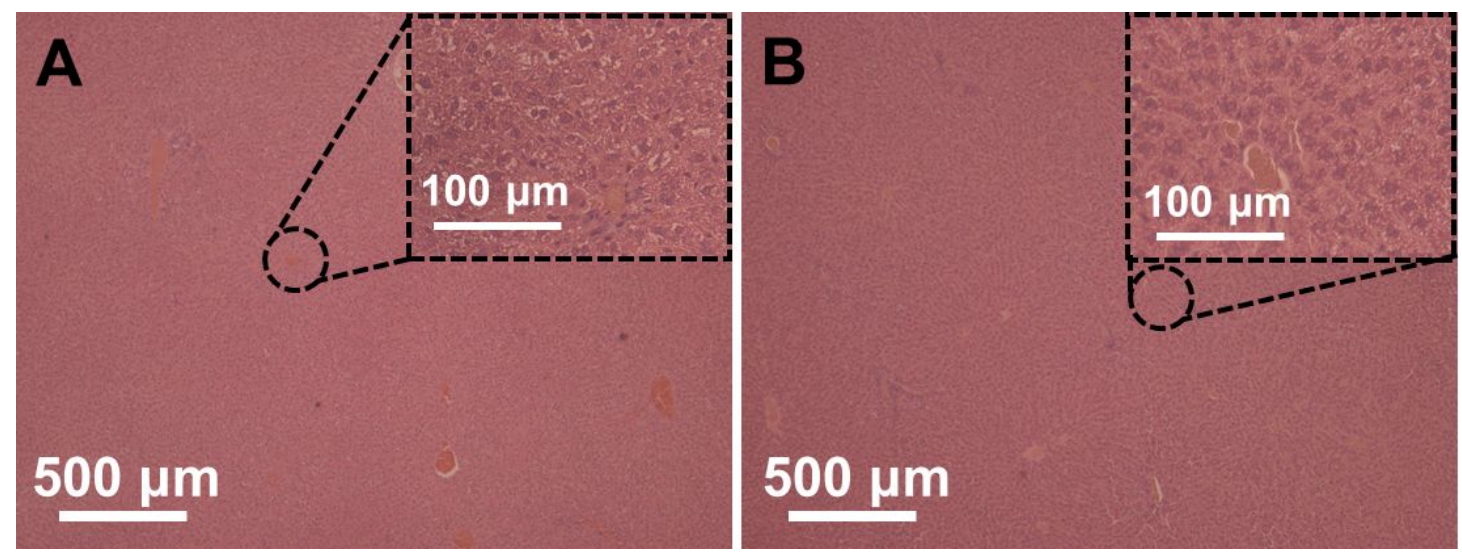

Fig. S7 H\&E staining of liver from normal SD rats liver (A) and injured SD rats after 12 days of treatment on the wound with the composite hydrogel dressing (B).

\section{References}

(1) He, M.; Wang, Q.; Wang, R.; Xie, Y.; Zhao, W.; Zhao, C. Design of Antibacterial Poly(Ether Sulfone) Membranes via Covalently Attaching Hydrogel Thin Layers Loaded with $\mathrm{Ag}$ Nanoparticles. ACS Appl. Mater. Interfaces 2017, 9, 15962-15974.

(2) Mao, C.; Xiang, Y.; Liu, X.; Cui, Z.; Yang, X.; Yeung, K. W. K.; Pan, H.; Wang, X.; Chu, P. K.; Wu S. Photo-Inspired Antibacterial Activity and Wound Healing Acceleration by Hydrogel Embedded with Ag/Ag@AgCl/ZnO Nanostructures. ACS Nano 2017, 11, 9010-9021.

(3) Hu, B.; Owh, C.; Chee, P. L.; Leow, W. R.; Liu, X.; Wu,Y. L.; Guo, P.; Loh, X. J.; Chen X. Supramolecular Hydrogels for Antimicrobial Therapy. Chem. Soc. Rev. 2018, 47, 6917-6929.

(4) Cui, Y.; Lu, C.; Chen, B.; Han, J.; Zhao, Y.; Xiao, Z.; Han, S.; Pan, J.; Dai, J. Restoration of Mandibular Bone Defects with Demineralized Bone Matrix Combined with Three-Dimensional Cultured Bone Marrow-Derived Mesenchymal Stem Cells in Minipig Models. J. Mater. Sci.-Mater. M. 2018, 29, 147.

(5) Ju, J.; Xu, X.; Xie, Y.; Guo, Y.; Cheng, Y.; Qian, H.; Yao, W. Inhibitory Effects of Cinnamon and Clove Essential Oils on Mold Growth on Baked Foods. Food Chem. 2018, 240, 850-855. 